\title{
Soberanía y ética en las relaciones internacionales: contextos superpuestos
}

\author{
CARLOS D. ESPÓSITO * \\ Universidad Autónoma de Madrid
}

1

En la teoría de las relaciones internacionales abundan los argumentos estratégicos basados en la defensa de intereses nacionales, que suelen ir acompaniados de pronuriciamientos sobre la inadecuación de los juicios morales respecto de la conducta de las naciones, sea porque los términos éticos no tienen el mismo significado en el contexto internacional o porque los juicios éticos son moralmente dañinos en sus consecuencias para las relaciones internacionales. Diplomáticos como George Kennan, filosófos políticos como Benedetto Croce, historiadores como Arthur Schlesinger o teóricos de relaciones internacionales como Hans Morgenthau, por citar algunos nombres significativos, están representados en ese grupo de pensadores. Es por esto que en varias ocasiones se ha sostenido que los estudios clásicos de teoría de las relaciones internacionales no abordan los temas éticos con la seriedad que merecen por diversas causas de endeble fundamento, entre las que cabe nombrar el escepticismo y el realismo (Cohen, 1984), aunque también algunas teorías postmodernas de la disciplina son susceptibles de la misma crítica (Frost, 1996).

Sin embargo, en los últimos años esa situación ha cambiado radicalmente por motivos teóricos y empíricos. En el campo teórico, en la década de 1970, comienzan a aparecer trabajos centrados en problemas de justificación, que coinciden con el resurgimiento de las discusiones sobre ética normativa tras la aparicion de la Teoria de la justicia de John Rawls y con diversos ataques al paradigma realista en la ética de las relaciones inter- nacionales (p. ej., Beitz, 1979). Además, en el terreno práctico, la necesidad de una discusión profunda de los problemas internacionales desde un punto de vista ético-normativo se demuestra cada vez más notoria si tenemos en cuenta el tipo de casos ante los que nos encontramos y el contexto en el que se producen. Respecto de este último, llama la atención inmediatamente la transformación internacional que se ha producido en las últimas décadas y que hacen no ya conveniente sino necesario hablar con nuevos términos como glohalización. Con razón se ha criticado este concepto por su ambigüedad, ya que puede describir «cualquier cosa desde Internet a una hamburguesas y que con frecuencia es aun eufemismo cortés para la continua americanización de los gustos consumistas y de las prácticas culturales" (Strange, 1996, p. xm). No obstante, globalización es un concepto útil porque, por un lado, nos permite una descripción más adecuada de una realidad internacional a la que le resulta imposible mantener sus problemas encerrados dentro de las fronteras de los Estados; pero que, por otro lado, nos alerta tambiên, y sin excluir el sentido normativo, de los nuevos análisis que se precisan para decidir cuestiones en las que no sólo hay elementos descriptivos, sino también preguntas sobre cómo se debe actuar en casos críticos como la inmigración por motivos políticos y económicos en los paises desarrollados, la protección del medio ambiente mundial en los países desarrollados y subdesarrollados, la utilización de energías altamente contaminantes y con grandes niveles de riesgo, junto a problemas más tradicionales como los

* El presente escrito se inscribe dentro de un proyecto de investigación, financiado por la DGICYT, del Programa Sectorial de Promoción del Conocimicnto (núm. PB 94-0193). 
reclamos de autodeterminación tendentes a la creación de nuevos Estados, la intervención por razones de humanidad en casos de grave violación de derechos humanos, los deberes positivos ${ }^{1}$ de los Estados frente a tragedias como el hambre o la falta de atención médica mínima allende sus fronteras y tantos otros.

Los estudios dedicados al análisis de dichos temas en la teoria de las relaciones internacionales se denominan «teoría normativas. Este es un concepto de textura abierta y muy extendido en la disciplina, que nace como reacción, pero no necesariamente como alternativa excluyente, al dominio casi absoluto de los estudios empíricos de relaciones internacionales, basados principalmente en la idea de paradigmas. Brown, por ejemplo, dice que por teoría normativa de las relaciones internacionales se entiende $k e l$ cuerpo de trabajo que estudia la dimensión moral de las relaciones internacionales y las preguntas generales de significado e interpretación generadas por la disciplina». Su intención más básica es estudiar cla naturaleza ética de las relaciones entre comunidades/Estados, sea en el contexto de la vieja agenda, que se centraba en la violencia y la guerra, o de la nueva agenda, que mezcla csas preocupaciones tradicionales con los reclamos modernos por una justicia distributiva internacional» $(1992, p, 3)$.

El objeto de estas páginas es exponer brevemente algunas ideas en relación con el contexto mundial en que se producen los casos arriba mencionados frente a un concepto esencialmente incontestado (Walker, 1993, contra Krasner, 1993) en las relaciones internacionales, que trasciende ideologías, auges y caídas de poderes (Hinsley 1986), es decir, la soberania. La noción de soberanía es esencial por su carácter fundacional, apoyado en la idea de que la soberanía estatal es la unidad estructural básica de la sociedad intemacional (Bull, 1977). ¿Es la soberanía un obstáculo para los juicios normativos en la teoria de las relaciones intcrnacionales? ¿Tiene hoy el concepto de soberania el valor explicativo y justificador que tradicionalmente se le otorga? ¿Puede afirmarse que los valores unidos a la idea de globalización, derechos humanos y democratizacion han socavado la idea de soberanía como concepto fundacional? Mi intención es presentar una descripción de algunos problemas bastante generales relacionados con esas preguntas. Comenzaré por mostrar una definición tradicional de soberanía en las relaciones internacionales y sus relaciones con una determinada idea del estatuto moral del Estado. La intervención en los asuntos internos de otros Estados y el fenómeno de la organización intemacional de integración nos servirán de ejemplos para ver hasta qué punto se ha erosionado o transformado el carácter fundacional del concepto de soberanía.

\section{2}

Una preocupación primordial de la teoría normativa de relaciones internacionales es la discusión sobre el estatuto moral de los Estados. Es lógico que así sea por las derivaciones teóricas que tiene adoptar una posición determinada sobre este problema. Todas las tradiciones de ética internacional, orientadas por las consecuencias ${ }^{2} \circ$ por las normas, adoptan su propio punto de vista al respecto (Nardin y Mapel, 1992); no obstante, para simplificar la discusión es conveniente seguir la propuesta de Chris Brown (Brown, 1992; Rengger, 1996), quien divide a los autores en dos grandes categorias, cosmopolitas y comunitaristas, según el valor que adscriban a las comunidades/Estados frente a los individuos que los componen. Es notable que Brown se aparte de la idea bast ante extendida en la escuela inglesa de relaciones internacionales, según la cual la teoría de las rêlaciones internacionales es un discurso diferenciado de la filosofía política, afirmando en cambio que la teoría de las relaciones internacionales es filosofía política - argumento que comparto absolutamente aunque sólo sea por su efecto enriquecedor de la argumentación doctrinal- ${ }^{3}$. De ahí que este autor busque sus fuentes y soportes teóricos entre los filosofos políticos para recrear las visiones aludidas: entre los cosmopolitas Brown inclu- 
ye, por supuesto, a Kant, pero también al utilitarismo y al marxismo, porque todos tienden y comparten un cierto tipo de universalismo; entre los pensadores comunitaristas se encuentra Hegel, los defensores de doctrinas nacionalistas $y$, curiosamente, una interpretación de J. S. Mill. Esta lista se traslada al contexto discursivo actual incorporando a Beitz del lado cosmopolita y a Walzer, Frost o Kymlicka del lado comunitarista.

La fuente de autoridad, como ocurre en el contexto de otras clasificaciones (Nardin y Mapel, 1992, pp. 304-305), es esencial en esta distinción: los cosmopolitas están dispuestos a aceptar razonamientos consecuencialistas, pero siempre respetando ciertos limites impuestos por reglas de conducta universales reconducibles a ideas de humanidad, dignidad o crueldad; en cambio, los comunitaristas, aun aceptando la existencia de algunas normas generales, rechazan la posibilidad de que esas normas surjan desde un espacio situado fuera de la comunidad. En consecuencia, para los cosmopolitas el estatuto moral del Estado no puede constituir por sí mismo una justificación para violar normas de conducta universalmente aceptadas, mientras que para los comunitaristas la carga de la prueba en el razonamiento se trasiada al ámbito interno, reaccionando, en principio, contrariamente a cualquier limitación externa nacida fuera de la comunidad misma. Un argumento ya clásico en este último sentido de la base moral de la autonomía del Estado se debe a Michael Walzer, quien en su Just and Unjust Wars, partiendo de un paradigma legalista compuesto por el principio de no intervención, la criminalización de la agresión y la legítima defensa, sostuvo que los derechos morales de las comunidades políticas pueden ser defendidos por los medios necesarios, incluyendo el recurso a medios que fuera de un contexto circunstancial de emergencia suprema sean considerados ilegales (Walzer, 1977 y 1980; crítica de Luban, 1980a y 1980b).

La idea de soberanía no es idéntica al problema planteado por el estatuto moral del Estado, pero se puede superponer a la discusión anterior, porque las pretensiones de todos los Estados respecto de una autonomía mínima o máxima, positiva o negativa, interna y externa, jurídica y política, está íntimamente ligada a la historia de los Estados soberanos en un tipo de sociedad internacional que tiene su origen en el siglo xvu con la división y secularización de los nuevos Estados que se consolidan como idea e institución politica tras la Paz de Westfalia en 1648. En efecto, en un sentido, el concepto de soberanía significa "una determinación de restringir los imperativos de la conciencia moral individual, anclados en creencias religiosas, y acordar, en cambio, prioridad a los requerimientos de la coexistencia pacífica dentro de un espacio político dadom (Forsyth, 1992, p. 25). La supremacía del orden sobre la justicia que surge de esa determinación, cuya consecuencia es elevar a la soberanía a una especie de constitución de las relaciones internacionales (Philpott, 1995, p. 353) y en todo caso a una "norma asentada de las relaciones internacionales (Frost, 1996), supone la vigencia de un sistema de relaciones internacionales gobernado por un "modelo de soberanía» que tiene al Estado como actor principal de las relaciones internacionales (p. ej., Carrillo Salcedo, 1995).

El modelo de Estados soberanos y las posibles concepciones de la idea de soberanía han sido objeto de gran cantidad de estudios clásicos; sin embargo, hoy asistimos a una especie de explosión del número de trabajos dedicados a su análisis desde diversos puntos de vista que confluyen en las ideas de autoridad, población y territorio ${ }^{4}$. Desde el punto de vista realista, por ejemplo, la soberania está representada por la existencia de «un poder centralizado que ejerce su autoridad legislativa y ejecutiva dentro de un cierto territorio» (Morgenthau, 1967, p. 299). Esta posición combina un monopolio de la fuerza de tipo kelseniano junto a un concepto de anarquia en el ámbito internacional, que evidencian dos dimensiones del concepto de soberanía: la doméstica y la internacional. La comprensión bidimensional de la soberania ha tenido muchas veces efectos pacificadores, como los que pueden deducirse de las consecuencias de la descripción de Forsyth, cita- 
da en el párrafo anterior, y efectos perversos, como los que se derivan de la «imagen de un mundo social donde la principal amenaza a la vida, la libertad y la felicidad de los individuos proviene de afuera, donde una sociedad bien regulada necesita ser protegida de incursiones externas por el poder del soberano, donde el orden y la justicia se sostienen internamente en una comunidad moral, pero son amenazadas externamente por la violencia cxtranjera" (Brown, 1992, p. 130). Esta imagen esconde en gran medida formas de opresión que no se corresponden con la mera guerra entre Estados soberanos y favorece, en ocasiones, la construcción de barreras para justificar acciones que no soportan el peso del carácter universal de los juicios éticos.

Fl concepto de soberania, por tanto, puede reconducirse a una determinada idea de autoridad legitima (Philpott, 1995) y se describe como la «institucionalización de la autoridad pública dentro de dominios jurisdiccionales mutuamente excluyentes* (Ruggie, 1986, p. 143). De ahí que en cualquier definición de sus contenidos irreductibles aparezca íntimamente unido a la idea de no intervención. Este hecho ha generado investigaciones para demostrar no sólo cómo funciona el concepto de sobcranía, sino también para indagar en su significado a través de su contracara, la prohibición de intervención, para comprobar los ejercicios de representación (Foucault), como pueden ser el rey o el pueblo, y de simulaciốn (Baudrillard) con que se llenan de referencias autoritativas a la soberanía en relación con un gobiemo o un pueblo (Weber, 1995). Asimismo, el principio de no intervención ha sido interpretado como el aspecto negativo del concepto de soberanía, haciendo uso de un esquema de análisis paralelo al que Isaiah Berlin ideara para describir distintos tipos de libertades (Jackson, 1990). Por estos motivos, el principio de no intervención nos puede servir como un buen ejemplo para tener más elementos de juicio sobre la erosión o transformación de la idea de soberanía.
En el prefacio de Intervention in World Politics (1984), Hedley Bull se preguntaba con agudeza cuál era el puesto de la intervención en la política internacional contemporánea; si existe una inconsecuencia entre la norma y los hechos que convierten a la primera en una regla carente de virtualidad $o$, por el contrario, es un principio vital de la estructura normativa internacional, y si la prohibición conserva su relevancia como un principio esencial para el orden mundial, cómo debe ser formulada, modificada o adaptada para responder a las circunstancias de nuestro tiempo. Tras repasar las contribuciones de los autores que participaron en aquella abra, Bull concluye que la intervención es un dato estructural de la sociedad de naciones, que viene impuesto por la existencia de grandes desigualdades de poder entre los Estados. Sin embargo, Bull sostiene que mientras la sociedad internacional siga estando basada en el principio de soberanía e independencia de los Estados, la regla que prescribe la prohibición de intervenir en la jurisdicción de otros Estados conserva su vigencia. Este principio, junto a la idea de soberanía, es también una norma asentada de las relaciones internacionales, porque requieren una justificación especial para ser rechazadas o no reconocidas (Frost, 1996). Pero aun aceptando que la proscripción de intervenir en los asuntos de otros Estados es una norma fundamental del orden mundial, es discutible cuál debe ser su contenido y sus excepciones para adecuarse a las necesidades del mundo actual.

El principio de no intervención ha sido tradicionalmente construido como una prohibición eminentemente jurídica que conviene tener en cuenta por su valor justificatorio y también por lo que representa dentro la tradición ética del Derecho internacional (Forsyth, 1992). La mayoria de los juristas españoles (p. ej., Carrillo Salcedo, 1976, p. 102), además del artículo 2.7 de la Carta de las Naciones Unidas, recurren a la fórmula de la resolución de la Asamblea General de las Naciones Unidas, que contiene la Declaración relativa a los principios del Derecho internacional referentes a las relaciones de 
amistad y cooperación de los Estados de conformidad con la Carta de las Naciones Unidas [Res. AGNU 2625 (XXV), de 24 de octubre de 1970 ] para realizar un análisis del principio de no intervención. Esta resolución contiene una enunciación muy amplia de la prohibición: se condena todo tipo de intervención estatal, sea directa o indirecta, y sea cual fuere el motivo, en los asuntos internos o externos de cualquier otro Estado. Las conductas subsumidas bajo el concepto de no intervención, unidas al rechazo de la intervención unilateral de los Estados realizada en la sentencia de la Corte Internacional de Justicia sobre las actividades militares y paramilitares en y contra Nicaragua (1986) da como resultado una norma de prohibición de gran alcance. Sin embargo, esta decisión de la Corte Internacional de Justicia merece ciertas precisiones, porque la Corte ha mantenido y fortalecido el principio de no intervención, pero también lo ha delimitado al establecer que la prohibición afecta a la jurisdicción doméstica de los Estados. En concordancia, la Corte ha dicho que la intervención prohibida debe recaer sobre materias comprendidas dentro de las esferas de libre decisión de los Estados, es decir, la elección de su sistema político, económico, social y cultural y la formulación de su política exterior. Además, en ese mismo sitio se exige que la intervención se realice por medios de cuerción respecto de esas esferas de libre discreción. Todo esto nos lleva a preguntarnos por los diferentes tipos de intervención.

Hay intervenciones unilaterales y colectivas, intervenciones humanitarias, intervenciones por invitación, intervenciones económicas, intervenciones que implican el uso de la fuerza. Existe un acuerdo general en que la intervención prohibida por el Derecho internacional es la acción coercitiva, decidida unilateralmente por un Estado, con el fin de inmiscuirse en los asuntos de la jurisdicción intema de otros Estados. Algunos estudiosos le dan más alcance a la prohibición y otros, aunque pocos, incluso menos, pero ese núcleo normativo conservará su vigencia mientras no cambie el orden mundial construido sobre la base de los Estados soberanos.
No obstante lo dicho, es necesario examinar el problema de las distintos grados de legitimidad de los diferentes tipos de intervención. En este sentido, una intervención unilateral no posee ni puede poseer el grado de legitimidad que tiene una intervención colectiva, que ha pasado por el tamiz de la discusión y la negociación antes de ser ejecutada, pero que además refleja un consenso entre Estados que será más relevante cuanto más mayoritario. Lo apuntado respecto a este tipo de intervención no agota sus diferemcias valorativas, porque el devenir histórico ha demostrado que las intervenciones colectivas comportan unas características que la hacen más proclive a que la consideremos con buenos ojos en el sistema interestatal: me refiero a los medios, normalmente más débiles, y a los fines, necesariamente más consensuados, que pueden adscribirse a este tipo de intervenciones (Luard, 1984). La conclusión a la que llegamos es que el ideal de la intervención legítima pasa por una adecuada institucionalización internacional que cree las condiciones para decidir tales acciones en un marco de legalidad y legitimidad.

Volviendo al problema de la erosión o transformación del concepto de soberania, últimamente algunos autores citan como excepciones al principio de no intervención a la defensa de la democracia, los derechos humanos y la protección del medio ambiente. Respccto de la democratización, desde el final de la Guerra Fría se alzan voces que hablan de la democracia como un nuevo principio de legitimidad interna en la sociedad internacional (Franck, 1992). Como nos ha demostrado la historia, una sociedad internacional homogénea, es decir, una sociedad internacional con un único principio de legitimidad interna, no ha sido ajena a las intervenciones, con lo cual esta condición no ha actuado siempre como un freno frente a las intervenciones. Un ejemplo de ello son las intervenciones dinásticas del siglo XIX (Hoffmann, 1984). Sin embargo, la cuestión es saber si un principio de legitimidad interna legitima, a su vez, una intervención externa para reponer el estado de cosas, esto es, un gobierno elegido democráticamente. Este jui- 
cio, por supuesto, dependerá de muchas variables. Por ejemplo, si comparamos la intervención de EEUU en Panamá en 1989 y la intervención en Haití de 1994, comprobaremos que ambas intervenciones tenian entre sus objetivos fundamentales la restauración de la democracia. No obstante, mientras que la primera de ellas fue decidida unilateralmente por EEUU y condenada como una violación flagrante del Derecho internacional (Res. AGNU 44/240, de 29 de dicicmbre de 1989), la segunda, aunque también implementada por el mismo país, tuvo el beneplácito del Consejo de Seguridad de las Naciones Unidas, que autoriz6 la operación (Res. 940, de 31 de julio de 1994). Estos casos indican una tendencia a considerar legítimas las decisiones institucionales sobre intervención. Pero incluso éstas tienen problemas gravísimos, debido fundamentalmente a la pobre institucionalización del sistema internacional y a las acusaciones de etnocentrismo que necesariamente van a estar presentes en cualquier discusión sobre el tema. Esta última crítica se hace más fuerte en relación con los derechos humanos y las concepciones sobre las personas y su dignidad.

En efecto, en materia de derechos humanos es muy difícil adoptar un punto de vista absoluto en favor o en contra de la intervención, porque en demasiadas ocasiones se ha utilizado este noble motivo con fines espúreos. Es por cso que muchos autores rcchazan esta idea con fundamento en las diferencias de poder entre los Estados. Aquí solamente haré dos tipos de consideraciones en torno al tema: primero, respecto de los derechos humanos como Derecho internacional positivo, y segundo, en relacion con las razones para apoyar o no una intervención humanitaria. En cuanto al valor juridico de los derechos humanos se debe destacar el hecho de que ciertos derechos humanos son obligatorios según el Derecho internacional (Henkin, 1987), ya sea porque su fuerza proviene de tratados internacionales o porque son normas de Derecho internacional general y, por consiguiente, caen fuera del ámbito afectado por la jurisdicción doméstica de los Estados. En consecuencia, el principio de no intervención no sería un obstáculo para intervenir en un Fstado que viola sistemáticamente y gravemente los derechos humanos de sus habitantes. En la teoría, según se adopte un punto de vista más cercano al cosmopolitismo o al comunitarismo, se hacen diferencias sobre el grado de gravedad de la violación de derechos humanos que justificaria una intervención: para algunos autores sólo hace falta una violación seria de derechos humanos (Tesón, 1988), otros exigen violaciones gravísimas, como el genocidio (Walzer, 1977 y 1994). Todo esto me lleva a la segunda cuestión, porque dado que existen razones muy sólidas para actuar colectiva $\mathrm{e}$ institucionalmente contra las violaciones sistemáticas de derechos humanos, se deben plantear las razones por las cuales rechazariamos una intervención humanitaria de esas características. Mi respuesta aquí no depende de la relatividad moral ni de la diversidad cultural, porque éstas no pueden servir para justificar la violación de derechos humanos básicos que caigan dentro de un consenso "tenue» (para utilizar la terminología de Walzer), sino que depende de razones prudenciales ligadas a la efectividad y a las consecuencias de la intervención.

En relación con la protección del medio ambiente, no es ninguna novedad para nadie que el tema constituye hoy una de las preocupaciones fundamentales de la humanidad en un sentido que supera las fronteras estatales. Al hilo de esta realidad se ha ido generando un concepto de sociedad de riesgos (Beck, 1986) cuyas consecuencias no están más vinculadas al lugar donde se generan, sino que afectan a la vida en toda la tierra y ciertamente en todas sus formas de manifestación; son riesgos que tienen una determinada globalidad, cualidad que socava en gran medida el principio clásico de no intervención porque afectan a la sociedad civil internacional (Rosenau y Czempiel, 1992; Peterson, 1992). El desafío es buscar las instituciones globales que mejor y más cficazmente puedan cumplir con el objetivo de la protección medioambiental, aunque seguramente deban seguir contando con los Estados para poder llevar adelante sus proyectos, ya 
que muchas veces no cuentan con el poder necesario para ejecutarlos (Haas/Keohane/Levy, 1993).

\section{4}

La soberanía no sólo se ha visto afectada en las prerrogativas que otorga a los Estados, sino también en su posición frente a otros actores legitimos de las relaciones internacionales, como son claramente las organizaciones internacionales, y menos claramente algunas comunidades políticas que deben defender sus derechos como actores demediados o incluso sin voz en la escena internacional; todos compiten (aunque también cooperan) con el Estado por un puesto como aclores legitimos de las relaciones internacionales.

Sobre todo si pensamos en supuestos de integración, esas organizaciones internacionales comportan un debilitamiento de las competencias estatales clásicas. El mejor ejemplo es la Unión Europea. En efecto, aunque la naturaleza de la Unión sea objeto de debate, especialmente entre aquellos que desean verla como un Estado federal con Bruselas (o iLuxemburgo!) como la «nueva Romas ${ }^{5}$ o como una mera organización intemacional de integración en la que los Estados miembros son los «señores de los Tratados», măs allá de ese debate la realidad indica que la Unión opera en algún lugar entre esos dos extremos y tiene una dinámica interna que viene erosionando y transformando la autoridad del Estado-nación europeo en su sentido más tradicional.

Es relativamente sencillo desde el punto de vista de los ciudadanos europeos observar esa realidad en aquellos ámbitos normativos donde los Estados miembros han delegado competencias en exclusividad a las Comunidades Europeas por la virtualidad que a las normas de Derecho comunitario les insufla la operatividad de los principios de eficacia directa y primacia del Derecho comunitario, conjuntamente con la posición del Tribunal de Justicia. Pero también se aprecia un fenómeno similar en áreas que transgreden la autoridad tradicional del Estado por sí mismas, como por ejemplo el fenómeno de la «sociedad en movimiento", del que forma parte la inmigración. En efecto, el caso europeo permite apreciar con nitidez el proceso de cambio ocurrido a raíz de los flujos migratorios económicos o políticos. Esto es así porque la creación de un metcado único europeo, exento en teoría del problema de las fronteras, donde puedan circular libremente los capitales y también las personas, aunque con mayor dificultad, ha tenido como contrapartida la preocupación por el desarrollo de «medidas compensatorias» que permitan controlar más y mejor la inmigración en Europa. En este sentido, es evidente que la autoridad tradicional del Estado soberano se ve amenazada por un problema transnacional, razón por la cual se diseñan medios para hacer converger un sistema de «gobierno internacional» en relación con la inmigración como forma defensiva de controlar un poder que se escapa de Ias manos de la soberanía tradicional (Mitchell y Russell, 1996). Esto es así porque las personas que tienen un estatuto personal defïnido por la descolonización, o por grandes cambios demográficos o por flujos migratorios, suponen una alternativa. y una amenaza para la autoridad tradicional del Estado (p. ej., Said, 1993).

\section{5}

La soberanía de los Estados y la ética, utilizando la expresiôn de Rawls, pertenecen a contextos superpuestos. Es notorio que desde la ética, toda aproximación a la idea de soberanía entraña una crítica del mundo tal como es y, en consecuencia, un deseo de cambio (James, 1986, p. 257). Sin embargo, esos cambios no necesariamente deben tener como objetivo acabar totalmente con la idea de soberanía, se entienda como ficción lógicamente necesaria de una autoridad final y absoluta (Hinsley, 1986), como independencia constitucional (James, 1986), como libertad negativa (Jackson, 1990) o como construcción social (Biersteker/Weber, 1996). Incluso las teorias cosmopolitas más renom- 
bradas, como la que contiene La paz perpetua de Kant, hacen lugar y hasta favorecen la construcción de un sistema de relaciones internacionales sobre la base de Estados. El motivo de esta posición puede encontrar muchas y variadísimas tespuestas: porque el Estado garantiza los derechos individuales de la forma más efectiva; porque es indispensable para establecer una escala de valores; porque es el único medio idóneo para buscar el bien común de la humanidad; porque es la descripción más comprensiva del mundo; porque es una forma institucional que puede tener como fundamento un contrato social; porque representa la libre determinación de un pueblo; porque es parte de un sistema de autoridad jurídica; porque es útil desde un punto de vista táctico... (Nardin y Mapel, 1992 , p. 321). 1 o cierto es que en un mundo de alternativas imperfectas (Komesar, 1994) la soberanía, una vez desmitificada (Henkin, 1995, p. 8), puede servir tanto para obstaculizar como para ayudar a conseguir ciertos objetivos éticamente valiosos. Por un lado, como he sostenido en otro lugar, puede ser muy importante respetar las fronteras estatales en materia de ejercicio de competencias ejecutivas o policiales (Espósito, 1993), ya que de otra manera deberíamos renunciar a creencias y libertades muy arraigadas dentro de ciertos contextos culturales. En este sentido, aunque signifique una concesión al mundo, según Huntington (1996), o sólo se pueda justificar desde una postura «liberal irónica» à la Rorty, creo que es valioso seguir defendiendo a Rushdie y todo lo que su caso significa. Por otro lado, las críticas al modelo soberano y lo que tiene de «romance con el Estado-nación» (Luban, 1980) son lógicas y necesarias, ya que en muchas ocasiones un cambio de modelo favorecerá la consecución de fines cono una mayor igualdad, el pluralismo, la participación, la justicia económica mundial, la reducción de la opresión y de la crueldad, la protección del medio ambiente, el mantenimiento de la paz y la seguridad y tantos otros. Esa tarea demanda la adopción de otras bases para enfocar el problema de los derechos además del Estado, que no necesariamente implica excluir al
Estado ni hablar inmediatamente de «un mundo sin fronteras" (Ohmae, 1990). Esta es, por ejemplo, la propuesta de autores como Rengger (1996, p. 100), quien sostiene que la teoría política debería tomar en serio la globalización y descubrir los modos de sostener y/o crear los tipos de comunidad moral que los nuevos contcxtos demandan y que no necesariamente están en abierta contradicción con el Estado.

\section{REFERENCIAS}

Carrillo Salcedo (1976): Soberanía del Estado y Derecho internacional, 2." ed., Madrid, Tecnos.

- (1995): (ver bibliografía).

BEck, Ulrich (1986): Risikogesellschaft. Auf dem Weg in einer andere Moderne, Frankfurt, Suhrkamp.

Bertz, Charles (1978): Political Theory and Intemational Relations, Princeton, N. J., Princeton University Press.

Brown, Chris (1992): (ver bibliografia).

BuLl. Hedley (1984) (ed.): Intenvention in World Politics, Oxford, Blackwells.

- (1977): The Anarchical Society: A Study of Order in World Politics, Londres, Macmillan.

BURLEY, Anne-Marie Slaughter (1993): «International Law and International Relations Theory: A Dual Agenda», 87 American Journal of International Law, pp. 205-239.

BURLEY, A. M., y Mattli, W. (1993): «Europe Before the Court: A Political Theory of Legal Integration", 47 International Organization, pp. 41-76.

CoHen, Marshall (1984): «Moral Skepticism and International Relations", originalmente publicado en 13 Philosophy and Public Affairs y reimpreso en Beitz, Cohen, Scanlon y Simmons (eds.), Intemational Ethics, Princeton, Princeton University Press, 1985, pp. 3-50.

Espósito, Carlos (1993): «Bene captus, male detentus", 5 Estudios de Jurisprudencia pp. 7-27. 
Forsyth, Murray (1992): «The Tradition of International Law, en Nardin y Mapel (ver bibliografía).

Franck, Thomas (1992): «The Emergent Right to Democratic Governances, en 86 American Joumal of Intemational Law, pp. 46-91.

Garretr, Geoffrey (1992): «International Cooperation and Institutional Choice: the European Community's Internal Market», 46 International Organization, pp. 533-560.

- (1995): «The Polifics of Legal Integration in the European Union», 49 International Organization, pp. 171-181.

Hass, Peter M.; KeOHaNe, Robert O., y Levy, Marc A. (1993): Institutions for the Earth, Cambridge, Mass., The MIT Press.

HenkIN, Louis (1995): Intemational Law: Politics and Values, Dordrecht/Boston/ Londres: Martinus Nijhoff Publishers.

- (1987): «The International Bill of Rights: The Universal Declaration and the Covenants», en R. Bernhardt y J. A. Jolowics (eds.), Intemational Enforcement of Human Rights, Berlín, Springer Verlag, pp. 1-19.

Huntington, Samuel P. (1996): The Clash of Civilizations and the Remaking of World Order, Nueva York, Simon \& Schuster.

KoMESAR, Neil (1994): Imperfect Altematives. Choosing Institutions in Law, Economics, and Public Policy, Chicago y Londres, The University of Chicago Press.

KrasNer, Stephen D. (1993): «Westphalia and All Thats, en Judith Goldstein $y$ Robert $O$. Keohane, Ideas \& Foreign Policy, Ithaca, Cornell University Press.

LUARD, Evan: «Collective Intervention», en Bull, 1984, pp. 157-179.

LuBAN, David (1980a): «Just Wars and Human Rights», originalmente publicado en 9 Philosophy and Public Affairs y reimpreso en Beitz, Cohen, Scanlon y Simmons (eds.), International Ethics, Princeton, Princeton University Press, 1985, pp. 195-216.

- (1980a): «The Romance of the Nation-State», originalmente publicado en 9 Philosophy and Public Affairs, y reimpreso en Beitz, Cohen, Scanlon y Simmons (eds.), International Ethics, Princeton, Princeton University Press, 1985 , pp. 238-243.

MAPEl y NARdin (1992): (ver bibliografía). Mattli, Walter, y Slaughter, Anne-Marie (1995): a Law and Politics in the European Union: A Reply to Garret», 49 International Organization, pp. 183-190.

Mitchell, Mark, y Russell, Dave (1996): "Immigration, Citizenship and the Nation-State in the New Europe», en Brian Jenkins y Spyros A. Sofos, Nation and Identity in Contemporary Europe, Londres, Routledge.

OHмaE, Kenichi (1990): The Borderless World, Londres, Collins.

Peterson, M. J. (1992): «Transnational Activity, International Society and World Politics», 21, Millennium, pp. 371-388.

PhupotT, Daniel (1995): «Sovereignty: An Introducción and Brief History», 48, Journal of International Affairs, Winter, pp. 353-368.

Rawls, John (1955): "Two Concepts of Rules», 64, Philosophical Review, pp. 3-32.

RENGGER, N. J. (1996); «Moral Communities and World Politics: Political Theory Beyond/Against the State», en M. Wright (ver bibliografía).

Rosenau, James, y Czempiel, Ernst-Otto (eds.) (1992): Govemance Without Government: Order and Change in World Politics, Cambridge.

RugGie, J. G. (1986): «Continuity and Transformation in the World Polity: Toward a Neorealist Systhesis», en Robert Keohane (ed.), Neorealism and its Critics, Nueva York, Columbia University Press.

SAdD, Edward (1993): Imperialism and Culture, Nueva York, Knopf, últino capítulo (hay una reciente traducción castellana en editorial Anagrama).

Tesón, Fernando R. (1997): Humanitarian Intervention: An Inquiry into Law and Morality, 2. ed., Nueva York, Dobbs Ferry. WALKER, R. J. B. (1990): (ver bibliografía). WALZER, Michael (1977): Just and Unjust Wars: A Moral Argument With Historical Illustrations, Nueva York, Basic Books. - (1980): aThe Moral Standing of States: A Response to Four Critics", originalmen- 
te publicado en 9 Philosophy and Public Affairs y reimpreso en Bcitz, Cohen, Scanlon y Simmons (eds.), Intemational Ethics, Princeton, Princeton University Press, pp. 217-237.

\section{BIBLIOGRAFÍA}

1. Estudios recientes en torno al concepto de soberanta:

- Biersteker, Thomas J., y Weber, Cynthia (eds.) (1996): State Sovereignty as Social Construct, Cambridge, Cambridge University Press.

- Camilleri, Joseph A., y Falk, Jim (eds.) (1992): The End of Sovereignty? Aldeshot, Edward Elgar.

- Caporaso, James A. (ed.) (1990): The Elusive State: International and Comparative Perspectives, Newbury Park, Sage.

- Carnor, Martin (1984): The State and Political Theory, Princeton, N. J, Princeton University Press.

- Carrillo Salcedo, J. A. (1995): Sobetania de los Estados y derechos humanos en Derecho intemacional contemporáneo, Madrid, Tecnos, 1995.

- Evans, Peter, et al. (1995): Bringing the State Back In, Cambridge, Cambridge University Press.

- Ferrajoli, Luigi (1995): La sovranità nel mondo moderno, Piacenza, Anabasi, 1995.

- Hall, J. A., e Ikeneerry, G. J. (1989): The State, Minneapolis, University of Minnesota Press. Hay traducción castellana: El Estado, Madrid, Alianza Editorial.

- Heln, David (1989): Political Theory and the Modern State: Essays on State, Power and Democracy, Stanford, Stanford University Press.

- JACKSON, Robert H. (1990): Quasi-States: Sovereignty, Intemational Relations, and the Third World, Cambridge, Cambridge University Prcss.

- JAMES, Alan (1986): Sovereign Statehood: The Basis of International Society, Londres, Allen \& Unwin.

- WALKER, R. B. J. (1993): Inside/Outside: Intemational Relations as Political Theory, Cambridge, Cambridge University Press.
- Weber, Cynthia (1995): Simulating Sovereignty. Intervention, the State and Symbolic Exchange, Cambridge, Cambridge University Press.

2. Estudios recientes sobre teoria normativa de relaciones internacionales:

- Brilmayer, Lea (1989): Justifying International Acts, Nueva York, Cornell University Press.

- Brown, Chris (1992); Intemational Relations Theon: New Normative Approaches, Nueva York, Columbia University Press.

- Buchanax, Allen (1991): Secession: The Morality of Political Divorce, Boulder, Westview Press.

- ChILd, James (1986): Nuclear War: The Moral Dimension, New Brunswick, Transac tion Books.

- Elus, Anthony (1986): Eihics and International Relations, Manchester, Manchester University Press.

- FalK, Richard:

- Finnis, John; Boyle, J., y GRISEz, G. (1987): Nuclear Deterrence, Morality and Realism, Oxford, Clarendon Press.

- Frost, Mervyn (1996): Ethics in International Relations. A Constitutive Theory, Cambridge, Cambridge University Press.

- Giesen, Klaus-Gerd (1992): L'éthique des relations intemationales, Bruselas, Bruylant.

- KeAl, Paul (ed.) (1992): Ethics and Foreign Policy, Londres, Allen and Unwin.

- KRatochwil, Friedrich (1989): Rules, Norms and Decisions, Cambridge, Cambridge University Press.

- Linklater, A. (1990): Men and Citizens in the Theory of Intemational Relations, $2 .{ }^{\text {. }} \mathrm{ed}$, Londres, Macmillan.

- Nardin, Terry, y Mapel, David (eds.) (1992): Traditions of International Ethics, Cambridge, Cambridge University Press.

- NyE, Joseph (1986): Nuclear Erhics, Nueva York, The Free Press.

- Onuf, Nicholas (1992); World of Our Making: Rules and Rule in Social Theory and International Relations, Columbia, SC, University of South Carolina Press.

- PogGe, Thomas (1989): Realizing Rawls, Ithaca, Cornell University Press. 
- Vincent, John (1986): Human Rights and International Relations, Cambridge, Cambridge University Press.

- WALZER, Micheal (1994): Thick and Thin: Moral Argument at Home and Abroad, Notre Dame, University of Notre Dame
Press. Hay traducción española de Rafael del Águila, Madrid, Alianza Editorial, 1996. - WRIGHT, Moorhead (ed.) (1996): Morality and Intemational Relations. Concepts and Issues, Aldeshot, Avebury.

\section{NOTAS}

3obre este tema ver los estudios de E. Garzón Vasidéz, Francisco Laporta y J. C. Bayón publicados en la revista $D O X A$ (1986).

${ }^{2}$ No todas las teorias orientadas por las consecuencias pueden ser llamadas consecuencialistas, ya que este último calificativo se aplica solo a aquellas teorias que evalúen los resultados desde un punto de vista impersonal o universal. Para ilustrar este punto sc puede recurrir a dos escuelas orientadas por las consecuencias, como son el utilitarismo y el realismo, aunque sólo la primera es consecuencialista (Mapel y Nardin, 1992, pp. 297-298).
${ }^{3}$ En el âmbito del Derecho internacional también es posible apreciar esta tendencia de búsqueda de leguajes comunes con la teoria de las relaciones internacionales. Ver, por ejemplo, Remiro Brotóns, 1996, Burlcy, 1993.

+ Ver una selección en la bibliografía.

' La expresión aparece en Krasner, 1993, p. 261. Muy interesante es el debate que al respecto se ha desarrollado en las páginas de International Organization entre Geoffrey Garret (1992, 1995), defendiendo una posición neorrealista de ta Unión Europea, y Burley/Mattli (1993) y Mattli/Slaughter (1995), adoptando un modelo de análisis neofuncionalista. 


\section{A S T R A G A L O}

CULTURA DE LA ARQUTTECTURA Y LA CIUDAD

CONSEJO DE DIRECCION:

ANTONIOF.AIBA/ROBERTO FERNANDEZ/EDUARDO SUBIRATS

ESPACIO Y GENERO

\section{ITTNERARIOS AL PARAISO}

José Luis Ramírez González

El espacio del género y el género del espacio

Nuria Fernández Moreno

La construcción cultural de los dominios masculino y fernenino. Espacios habitados, lugares no ocupados

Anna Vila i Nardi

Vicent Casals Costa

Elementos para una historia de las relaciones entre género y praxis ambiental. Itinerarios al paraiso

Carmen Pena López.

Estereotipos femeninos en la pintura. Palidas y esquirolas

Constanza Tobio

Zonifucación y diferencias del género

Carmen Gavira

Si las mujeres hicieran las casas...

Angelique Trachana

El carácter femenino de la arquitectura. Poesía y seducción

FORO ABIERTO

Geors Simme!

La rcasa)

Olivier Mongin, Benoît Chantre y Joël Roman

Debate

RESENAS DE LO PUBLICADO

Roberto Fernández

El largo adiós

RELATOS DE LO YA VISTO

Antonio Fernández-Alba

Nueva Forma o la lucidez de la agonía

POSFOLIO

Renate Mayntz

Progreso técnico, cambio de sociedad y clesarrollo de los grandes sistemas técnicos

INSTITUTO ESPAÑOL DE ARQUITECTURA

UNIVERSIDADES DE ALCALA Y VALLADOLID

Paseb de la Fstación, 10. Palacete Laredo

28807 ALCALA DE HENARES (Madrid - España) 